\title{
Article \\ Research on the Application Performance of the Helmholtz High-Efficiency Wave-Elimination Chamber in Gas Wave Tubes
}

\author{
Wutekuer Nuermaimaiti, Xuewu Liu *, Pengze Yan, Jiupeng Zou and Dapeng Hu \\ School of Chemical Engineering, Dalian University of Technology, Dalian 116024, China; \\ wutekuer@mail.dlut.edu.cn (W.N.); pengzeyolo@163.com (P.Y.); zoujiupeng@dlut.edu.cn (J.Z.); \\ Hudapeng@dlut.edu.cn (D.H.) \\ * Correspondence: liuxuewu@163.com
}

check for updates

Citation: Nuermaimaiti, W.; Liu, X.; Yan, P.; Zou, J.; Hu, D. Research on the Application Performance of the Helmholtz High-Efficiency

Wave-Elimination Chamber in Gas Wave Tubes. Energies 2022, 15, 882. https://doi.org/10.3390/en15030882

Academic Editor: Dmitry Eskin

Received: 3 December 2021

Accepted: 7 January 2022

Published: 26 January 2022

Publisher's Note: MDPI stays neutral with regard to jurisdictional claims in published maps and institutional affiliations.

Copyright: (c) 2022 by the authors. Licensee MDPI, Basel, Switzerland. This article is an open access article distributed under the terms and conditions of the Creative Commons Attribution (CC BY) license (https:// creativecommons.org/licenses/by/ $4.0 /)$.

\begin{abstract}
A gas wave refrigerator is a device that uses gas pressure to expand and refrigerate; the moving shock wave in the gas wave tube is the key to cooling. If the shock wave is reflected to the open mouth of the gas wave tube, it will heat the expanded refrigeration gas at the open end, severely reducing the refrigeration efficiency. In order to reduce this effect, a Helmholtz middle wave chamber-type gas wave tube is presented here. This study describes the performance of this structure in the gas wave refrigeration process. The refrigeration performance of the structure's gas wave tube use was evaluated experimentally and numerically using computational fluid dynamics (CFD). The function of the middle wave elimination chamber was studied and explained: the chamber turns the reflected shock wave back to the end section of the tube and converts part of the incoming shock wave to an expansion wave, which may counteract the reflected shock wave by escaping from wave elimination chamber. The experimental results showed that the structure increased refrigeration efficiency by about $2.9 \%(50 \mathrm{~Hz}), 2 \%(40 \mathrm{~Hz})$, and $2.2 \%(25 \mathrm{~Hz})$ at the corresponding ejection frequencies, and reduced the reflected shock wave intensity by $11 \%$, which proves the highefficiency energy dissipation performance and the significant increase in refrigeration efficiency of the Helmholtz-type gas wave tube.
\end{abstract}

Keywords: gas wave tube; wave-eliminating chamber; Helmholtz chamber; refrigeration efficiency

\section{Introduction}

Due to the high storage and transportation risk factors, the efficient use of pressure energy has long plagued researchers. Whether the pressure energy is converted from other energy or associated pressure energy, it must be used or converted in time to efficiently use its resources. A gas wave refrigerator (also called a thermal separator) is a piece of equipment that uses gas pressure to expand and refrigerate [1,2]. Its advantages include simple structure, low cost [3], convenient operation and maintenance, high pressure resistance, operation with liquid, low power consumption, etc. The basic principle is to inject high pressure gas from a rotating distributor (rotary type) or a wall jet oscillator (static type) into several gas wave tubes with initial openings in turn. The pulse jet formed will perform expansion work on the stagnant gas in the tube, and the enthalpy of the jet gas itself will be lowered and cooled [4].

Research on static gas wave refrigerators is still in the development stage. The air wave tube (also called the receiving tube) is closed at the end to eject air intermittently. A "contact surface" formed between the ejected air and the trapped air in the tube moves forward, and multiple compression waves are continuously generated until a positive shock wave is formed [2,5]. The shock wave sweeps the gas along the tube, increasing the temperature and pressure of the gas; the energy of the shock wave dissipates as it is transmitted to the gas through the tube wall, thereby greatly reducing the enthalpy and temperature of the incident gas (refrigeration) [6,7]. The forward movement of the shock wave continuously transfers energy to the original gas in the tube, thereby attenuating the 
shock wave. However, when the shock wave is not completely attenuated, it can move to the end of the tube and reflect back to the outlet of the gas wave tube. The reflected shock wave will have two adverse effects on gas refrigeration. The first is to heat the cold air that was refrigerated to be discharged, such that the temperature of the cold air rises again. The second is to increase the pressure of the jet inlet, increase the oscillator load, and reduce the incident pressure ratio. Both losses will directly reduce the efficiency of gas wave refrigeration.

Elimination the reflected shock wave is an important research direction to improve the efficiency of gas wave refrigeration. The conventional method is to lengthen the gas wave tube to reduce the shock wave such that it is unable to reflect; however, this wastes a significant amount of materials, causes vibration and condensate accumulation, and blocks the gas wave tube, making the effective tube length heavy and short. Fang et al. [8] proposed the addition of a shock absorption chamber with a limited volume at the end of each gas wave tube to dissipate and absorb the shock wave; the refrigeration efficiency was greatly improved, yet the fluctuation in the ejection frequency was still large. Hu et al. [9] proposed using multiple shock absorption cavities in series to further attenuate the reflected shock waves. Zou et al. $[10,11]$ experimentally studied the damping dissipation of wire mesh in the end of the gas wave tube, and Li et al. [12]. experimentally studied the shock wave with an orifice composite structure and achieved certain results. Dai et al. [13]. used CFD calculations to show that two composite structures can attenuate the shock wave to an acceptable level. In addition, Zhu [14], Li [15], and Liu et al. [16] studied the matching of the length of the gas wave tube with the ejection frequency, such that the gas wave tube can operate at a high-efficiency ejection frequency and reduce the impact of reflected shock waves. It is also possible to increase the heat exchange by changing the tube wall structure, increasing the energy dissipation in the tube, and reducing the reflected shock wave. For example, in the research of Al-Obaidi et al. [17-19], different dimple geometrical configurations with a combination of corrugated tubes and twisted tape were numerically investigated. The study found that vortices and rotational flow originated behind and near the dimple, twisted tape, and corrugation surfaces. The rotational flow and vortices promote mixing in flow between the thermal boundary layer and velocity boundary flow layer, thus increasing the heat transfer enhancement. This method can also improve refrigeration efficiency. Zou et al. [10,11] proposed moving the shock wave absorption chamber forward to a proper position behind the middle of the gas wave tube, creating a middle shock wave absorption chamber that was used to reduce the return of the reflected shock wave and fold the reflected shock wave back to the back section of the gas wave tube to accelerate its dissipation. The refrigeration efficiency was improved; however, there was significant incident energy loss in the fluid.

In a uniform straight gas wave tube, the reflected shock wave will maintain a high intensity and return to the exit position. If a wave-eliminating cavity is installed, the reflected shock wave can be attenuated by multiple reflections and energy dissipation in the cavity, and the intensity of the reflected returning shock wave will be significantly reduced, improving the cooling efficiency of a gas wave tube with the same tube length. In order to efficiently use the ejection pressure energy and reduce the reflected shock wave, one should also try to reconstruct the distribution and reflection of the wave system in the gas wave tube, improve the shock wave forward path, block the shock wave reflection, and increase the dissipation in the back section of the gas wave tube, thus improving shock wave attenuation. According to this idea, we proposed and studied the Helmholtz-type wave-elimination chamber structure, which was used to enhance the incident shock wave, prevent the return of the reflected shock wave, and fold the reflected shock wave back to the back section of the gas wave tube to accelerate energy dissipation and reduce the impact on the exit position of the reflected shock wave. Based on this, a new and efficient Helmholtz gas wave tube was developed. 


\section{Using CFD to Model the Chamber Geometry and Simulate the Flow Field of a Gas}

\section{Wave Tube}

\subsection{Creating a Chamber Geometry Model}

According to the previous analysis, to improve the efficiency of a static gas wave refrigerator it is necessary to increase the shock wave forward path, block the shock wave reflection, and increase the dissipation of the back section of the gas wave tube to promote shock wave attenuation. The proposed Helmholtz wave-elimination chamber structure is strictly symmetrical along the axis in geometry, and the three-dimensional model can be simplified to two-dimensional in the calculation. If the ejection frequency is controlled well, this cavity in the middle can make a small part of the shock wave that escapes from the left side of the cavity reflect the shock wave, which will meet the incident rightward expansion wave caused by the next ejection jet and the two will cancel out. Thus, the harm of the reflected shock waves is basically eliminated. Figure 1 shows the schematic diagram of the Helmholtz wave-elimination chamber.

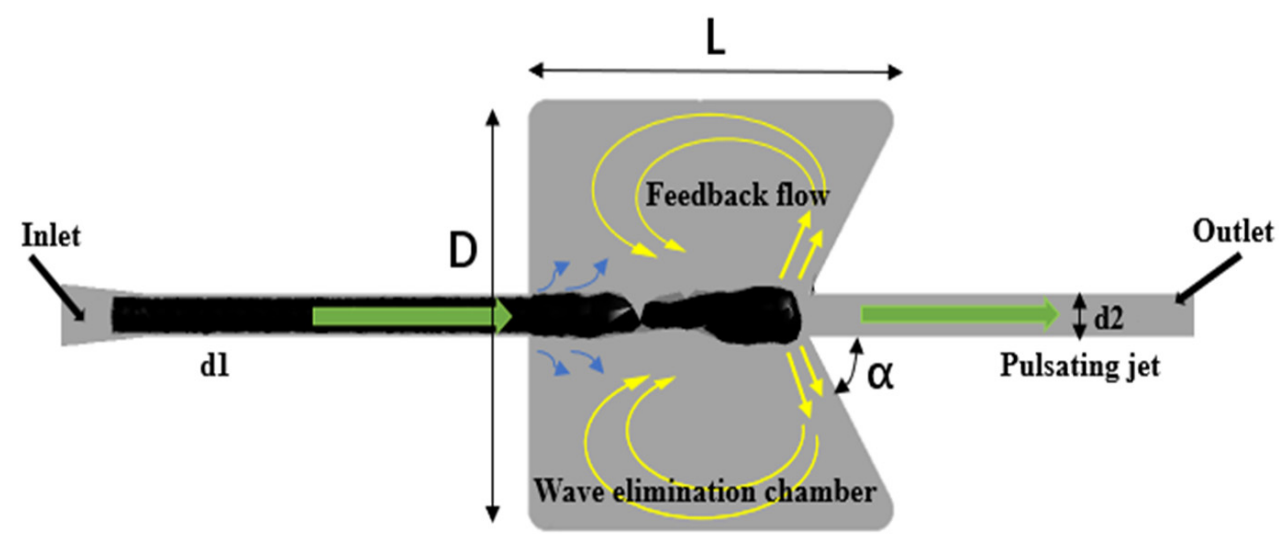

Figure 1. Schematic diagram of a Helmholtz wave-elimination chamber.

After the jet enters the chamber, the boundary layer disturbance is amplified by the resonance of the oscillating chamber to form an annular feedback flow vortex. The vortex ring further develops to form a large-area cavitation, blocking the continuity of the jet and forming a self-oscillating pulse jet.

Figure 2 shows the schematic diagram of a traditional wave-elimination chamber. Generally, after the jet enters the chamber, the boundary layer disturbance is amplified by the wave-elimination chamber and directly hits the wall of the vertical chamber. After a large amount of kinetic energy is lost, the feedback flow vortex is formed; however, the regular vortex ring cannot be completely formed, causing the jet to lose a significant amount of pressure.

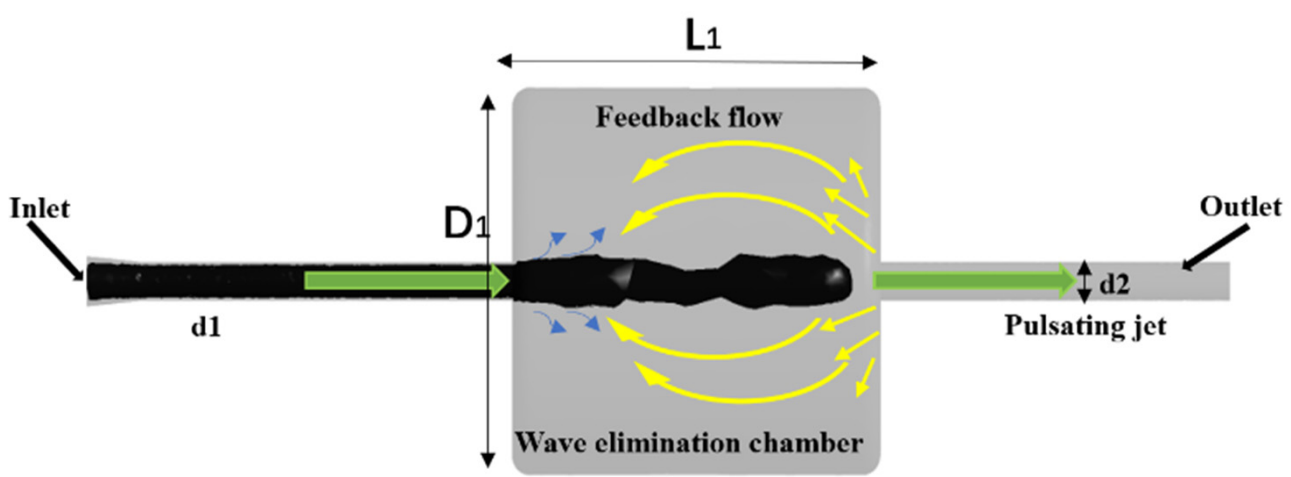

Figure 2. Schematic diagram of a traditional wave-elimination chamber. 
This geometric model adopts the Helmholtz wave-elimination chamber and traditional wave-elimination chamber comparison model. The dimensionless parameters of Helmholtz geometry were obtained from the related literature [20-22], and they can be used as a comparison with the traditional chamber. For comparison, these parameters are shown in Table 1.

Table 1. Comparison chamber parameters.

\begin{tabular}{ccc}
\hline Structure & $\begin{array}{c}\text { Helmholtz } \\
\text { Wave-Elimination Chamber } \\
(\mathbf{m m})\end{array}$ & $\begin{array}{c}\text { Traditional } \\
\text { Wave-Elimination Chamber } \\
(\mathbf{m m})\end{array}$ \\
\hline Entrance diameter $\mathrm{d}_{1}$ & 10 & 10 \\
Outlet diameter $\mathrm{d}_{2}$ & 10 & 10 \\
Chamber length L & 100 & 100 \\
Chamber diameter D & 100 & 100 \\
Inclination angle of posterior & $60^{\circ}$ & $90^{\circ}$ \\
chamber wall $\alpha$ & & \\
\hline
\end{tabular}

\subsection{Calculation Models and Boundary Conditions}

CFD was used to simulate a double-chamber gas wave tube with the traditional waveelimination chamber and the Helmholtz wave-elimination chamber, designed in the middle of the tube; an absorption chamber was also added at the ends of the two kinds of gas wave tubes. The two kinds of tube structures and the calculation mesh division are shown in Figure 3. Using a two-dimensional calculation model, a maximum mesh size of 0.2 $\mathrm{mm}$ was determined by independence analysis, and the mesh at the opening of the tube was densified.
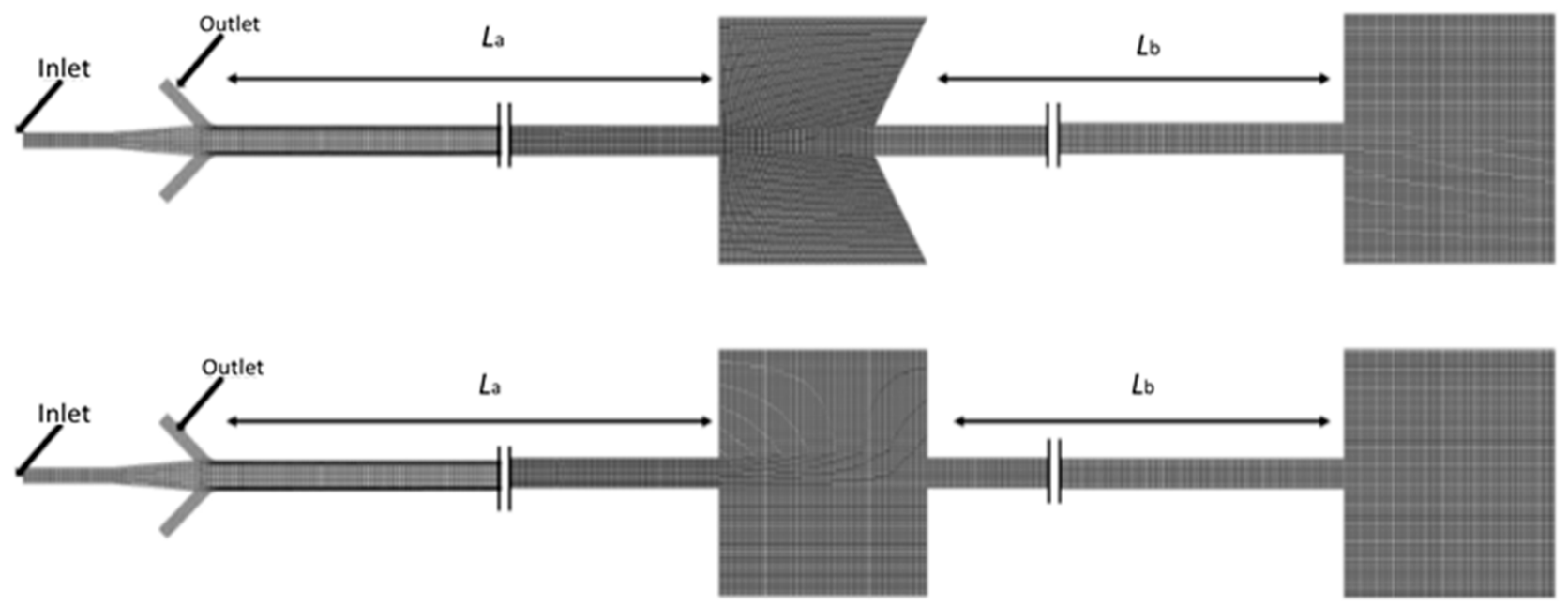

Figure 3. Structures and mesh of each type of gas wave tube.

The end absorption chamber was closed on the same side as the wave-elimination chamber; the front section of the middle wave-elimination chamber was $L_{\mathrm{a}}=2000 \mathrm{~mm}$, the rear section was $L_{\mathrm{b}}=1000 \mathrm{~mm}$, and the total length of the two kinds of tubes with the chamber was $3200 \mathrm{~mm}$. Since only the efficiency of the two gas wave tubes were compared, the pulse flow setting was realized by programming the pulsating intake function (UDF) in the simulation process. We used a trapezoidal wave to simulate the incident wave. As shown in Figure 4, the time for the rising and falling of the leading edge was $1 / 40 \mathrm{~s}$ of the period $\mathrm{T}$. 


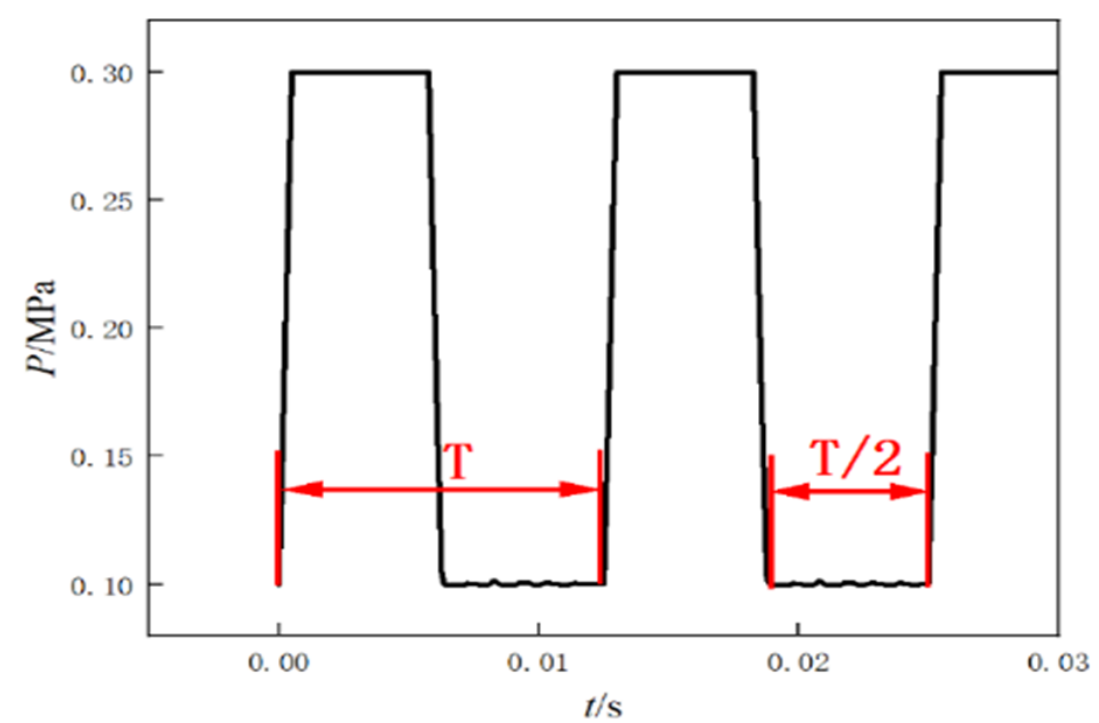

Figure 4. Trapezoidal total pressure waveform of external stimulation flow.

The pulse jet function (UDF) shown in Figure 4 is as follows:

$$
P_{A}=\left\{\begin{array}{lr}
P_{L}+\left[\left(P_{h}-P_{L}\right) / 0.0005\right] t & (0<t<T / 40) \\
P_{h} & (T / 40<t<19 T / 40) \\
P_{h}-\left[\left(P_{h}-P_{L}\right) / 0.0005\right](t-0.0095) & (19 T / 40<t<T / 2) \\
P_{L} & (T / 2<t<T)
\end{array}\right.
$$

where $t$ represents any time, $T$ is the oscillation period, $P_{\mathrm{A}}$ is the certain instantaneous excitation pressure value, $P_{\mathrm{L}}$ is the minimum pressure, and $P_{\mathrm{h}}$ is the maximum pressure.

The total pressure of the gas wave tube port in the ejection stage was $0.2 \mathrm{MPa}, 0.3 \mathrm{MPa}$, and $0.4 \mathrm{MPa}$, and the temperature was $300 \mathrm{~K}$, the cold air exhaust stage was subsonic flow, the static pressure value of the fixed nozzle was $0.1 \mathrm{MPa}$, and the corresponding expansion ratios $\varepsilon$ (the ratio of the total inlet pressure to the static outlet pressure) were 2, 3, and 4 , respectively, at fixed wall boundaries. The gas hypothesis conformed to the ideal gas state equation. The gas injection frequency $\mathrm{f}$ was $25 \mathrm{~Hz} \sim 80 \mathrm{~Hz}$ (in $5 \mathrm{~Hz}$ increments); the gas was assumed to conform to the ideal gas equation of state, and the viscosity was $1.789 \times 10^{-5} \mathrm{~kg} / \mathrm{m} \cdot \mathrm{s}$.

\subsection{Numerical Calculation Method}

The inlet of the gas wave tube pulsating jet is transonic, and it produces compressible and strong turbulent flow. The Reynolds average method for solving the time-averaged Navier-Stokes equation should be adopted, considering that the nozzle jet is turbulent and the turbulent flow is a highly non-linear and complex flow. The simulation method adopts the two-equation realizable $\mathrm{k}-\varepsilon$ turbulence model, and the finite volume method is used to discretize the control equations [23]. The diffusion term selects a central difference scheme with high computational efficiency and second-order accuracy, which converges quickly. The convection term is anisotropic. In order to avoid numerical oscillations, the MUSCL (Monotonic Upstream-centered Scheme for Conservation Laws) format of the Roe flux differential splitting in the upside-down style is used for discretization [24], and the second-order fully implicit time step is used for iteration [25].

\subsection{Refrigeration Efficiency of the Gas Wave Tube}

The refrigeration efficiency of the gas wave tube is the ratio of the actual enthalpy drop of the jet gas entering and exiting the gas wave tube to the ideal isentropic enthalpy 
drop. The specific heat of the ideal gas does not change with pressure, as shown in the following formula:

$$
\eta=\frac{C_{p} m\left(T_{\text {in }}-T_{\text {out }}\right)}{C_{p} m T_{\text {in }}\left[1-\left(\frac{P_{\text {out }}}{P_{\text {in }}}\right)^{\frac{k-1}{k}}\right]}=\frac{T_{\text {in }}-T_{\text {out }}}{T_{\text {in }}\left[1-\left(\frac{1}{\varepsilon}\right)^{\frac{k-1}{k}}\right]}
$$

where $k$ is the isentropic process index of the gas, $T_{\text {in }}$ is the total temperature at the inlet of the jet gas, and $T_{\text {out }}$ is the time-average total temperature at the outlet of the gas wave tube.

During the simulation process, the mass-weighted average total temperature $T_{\mathrm{av}}$ of the outlet section of the gas wave tube is monitored over time. When the change with time is stable (we selected the seventh period), the outlet section of the gas wave tube outlet section $T_{\mathrm{av}}$ and $\Delta t$, the product of the mass flux $\Delta m$, are accumulated and then divided by the integral value $\mathrm{m}$ of the total exhaust mass at the outlet of the gas wave tube; finally, the time-average total temperature $T_{\text {out }}$ of the gas at the outlet of the gas wave tube can be obtained.

\section{Flow Field Simulation and Results Analysis of the Two Kinds of Chambers}

For the three-dimensional simulation of the two cavities, the simulation conditions were as follows: the ejection frequency was $80 \mathrm{~Hz}$, the total pressure of pulsating ejection was $0.3 \mathrm{MPa}$, the temperature was $300 \mathrm{~K}$, the static pressure at the outlet stage was $0.1 \mathrm{MPa}$, and the corresponding expansion ratio $\varepsilon$ was 3 . Figure 5 shows a cloud diagram of the velocity vortex field changes inside two kinds of chambers. It can be seen from the figure that the jet in the Helmholtz-wave-elimination chamber begins to spread from the edge of the jet with time after ejection. When the time of reaching the back chamber wall is $t$, the edge of the jet collides with the back wall of the wave-elimination chamber, forming a tiny backflow. The backflow forms a vortex in the wave-elimination chamber, and the formation of this vortex will in turn disturb the edge shear layer of the jet, forming positive feedback, and finally strengthening the vortex flow inside the oscillation chamber and intercepting the continuous jet to form a self-oscillating jet. In the traditional waveelimination chamber, the time it takes for the jet to enter the chamber, the wave-elimination chamber to amplify the boundary layer disturbance, and the jet to directly hit the vertical chamber wall, is $t$. After a large amount of kinetic energy is lost, the feedback flow vortex is formed; however, the regular vortex ring cannot be completely formed, causing the jet to lose a significant amount of pressure.

Figure 6 shows the pressure changes at the outlet of the two chambers. The Helmholtz chamber excited a stable self-oscillating jet under a condition of small pressure loss. The total pressure of the Helmholtz wave-elimination chamber was much higher than the traditional wave-breaking chamber. The total pressure retention rate increased by $7 \%$. This shows that the forward collision of the jet in the traditional chamber consumes a great deal of energy. Reducing the jet pressure front jump speed and the slow pressure drop causes a large amount of energy loss when the jet collides with the back chamber wall and cannot be avoided. Therefore, the total outlet pressure is lower than in the Helmholtz chamber, which affects the refrigeration efficiency.

Figure 7 shows the outlet velocities of the two chambers. The Helmholtz waveelimination chamber jet velocity loss was significantly lower than that of the traditional wave-elimination chamber, and the velocity increased by about $50 \mathrm{~m} / \mathrm{s}$, which also doubled the amplitude of the jet's self-oscillation pulsation and increased the jet turbulence at the end of the gas wave tube, improving the energy dissipation efficiency. 

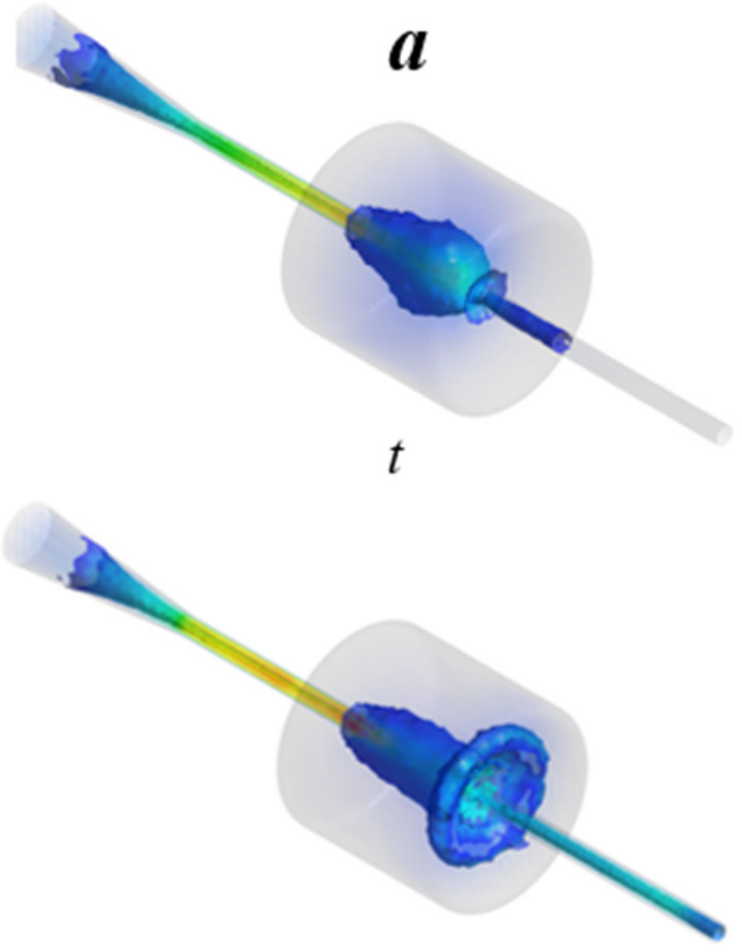

$t+0.4 m s$

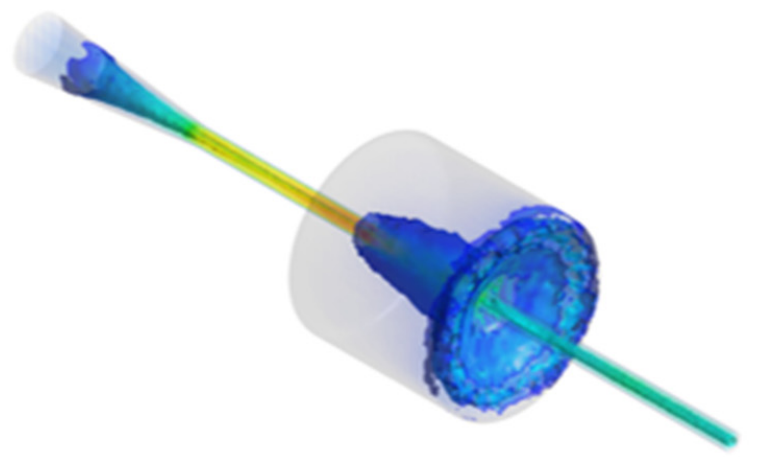

$t+0.8 \mathrm{~ms}$

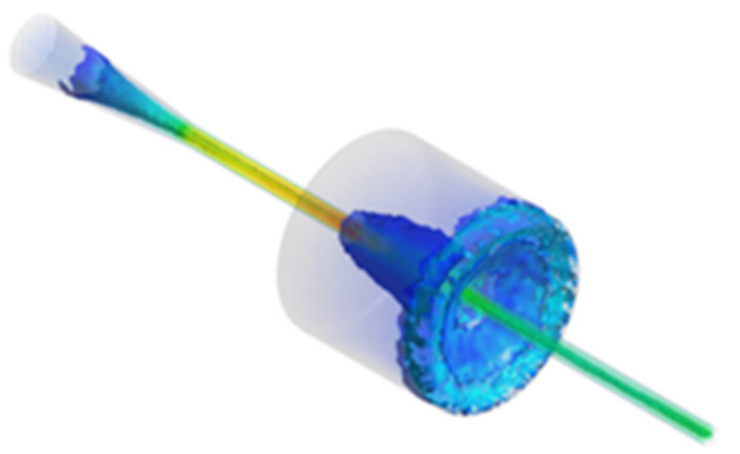

$t+1.2 \mathrm{~ms}$ b
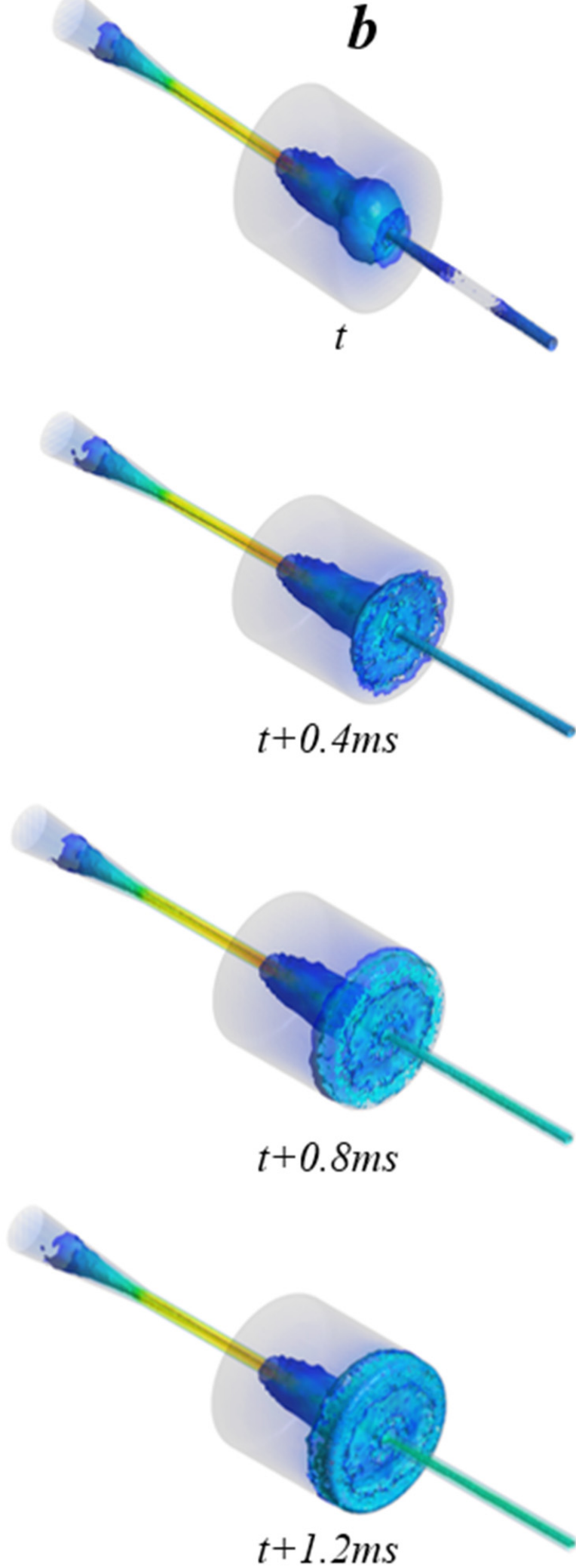

Figure 5. Variations of the velocity vortex field inside the two chambers: (a) Helmholtz waveelimination chamber; (b) traditional wave-elimination chamber. 


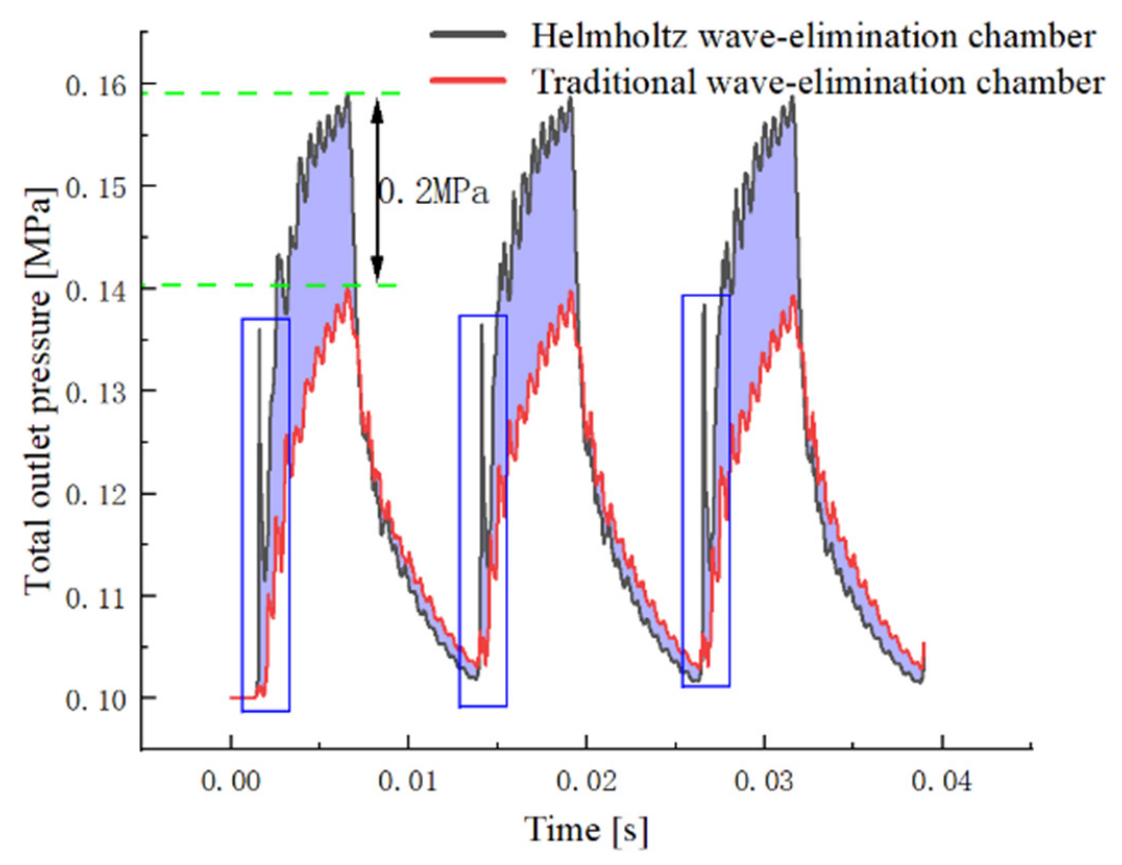

Figure 6. Outlet pressure of the two chambers.

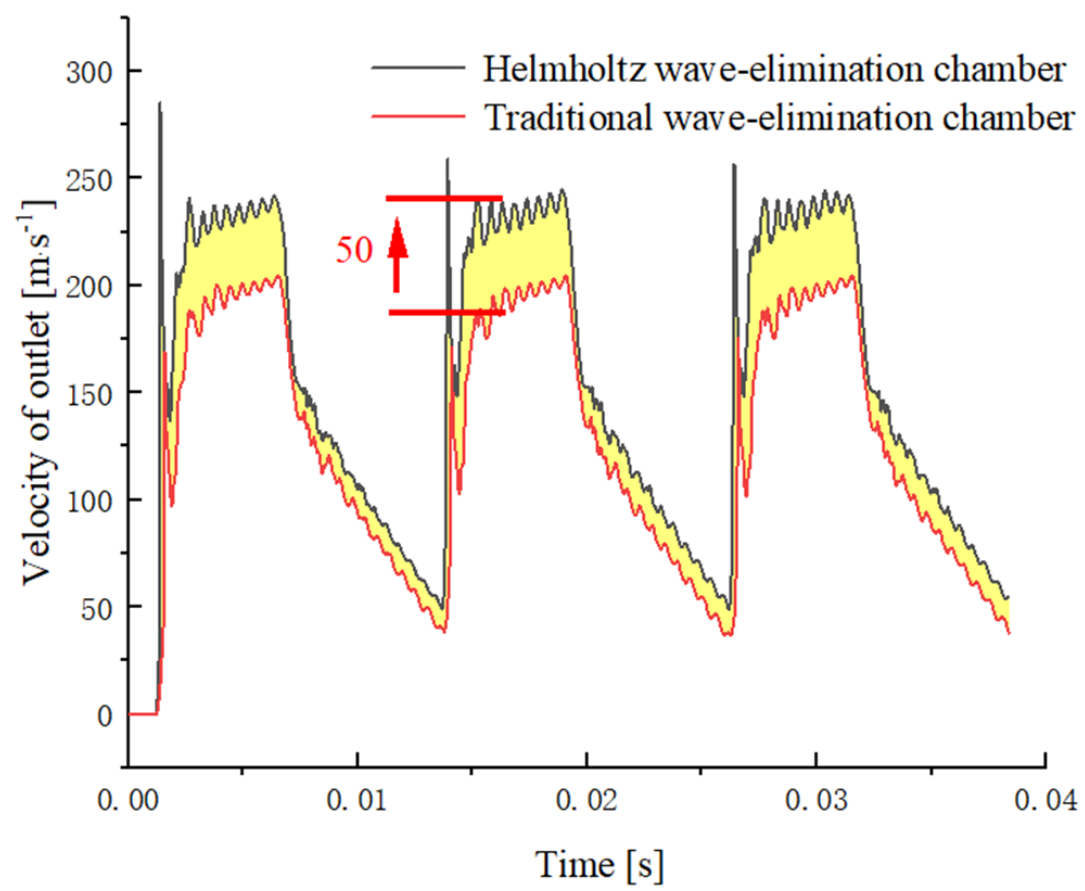

Figure 7. Velocity of the two chambers.

We observed the effect of the change in the expansion ratio on the outlet pressure of the Helmholtz wave-elimination chamber. When the pulsating ejection frequency was $80 \mathrm{~Hz}$, the total pressure of pulsating ejection was $0.2 \mathrm{MPa}, 0.3 \mathrm{MPa}$, and $0.4 \mathrm{MPa}$, and the temperature was $300 \mathrm{~K}$, the cold air exhaust stage was subsonic flow, the static pressure value of the fixed nozzle was $0.1 \mathrm{MPa}$, and the corresponding expansion ratios $\varepsilon$ were 2,3 , and 4 , respectively. It can be seen from Figure 8 that reducing the expansion ratio slows down the pressure front jump speed and pressure drop speed. Therefore, the Helmholtz chamber would have a better effect on the end jet at a high expansion ratio. 


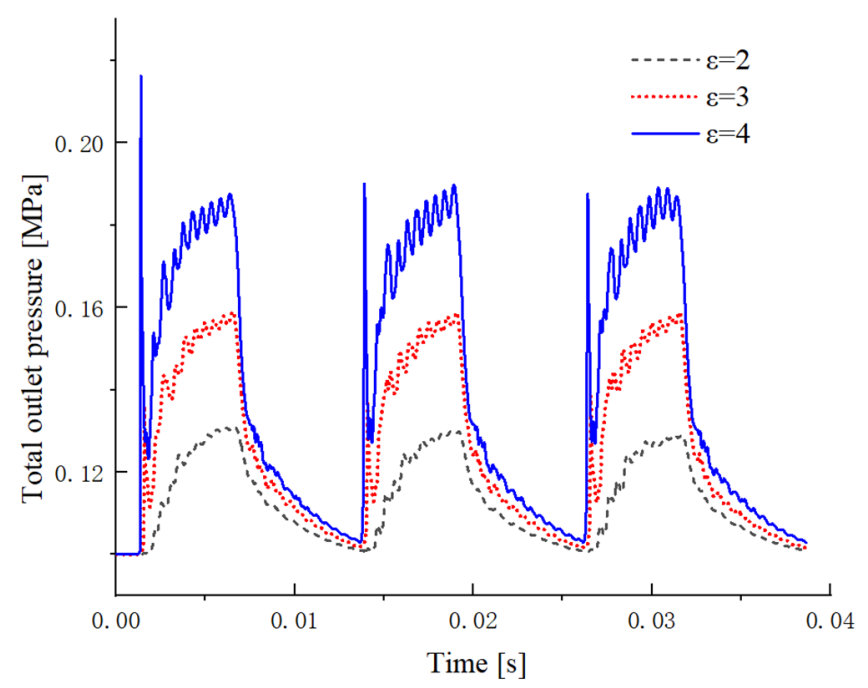

Figure 8. Outlet pressure change under different expansion ratios.

\section{Comparison and Analysis of the Efficiency of the Two Kinds of Gas Wave Tube Refrigeration}

Through the method of two-dimensional numerical simulation, the calculation was carried out by combining different frequencies of pulsating jets with a closed gas wave tube structure. The intensity of the shock wave is defined as the ratio of the total gas pressure in the gas wave tube to the static pressure at the outlet. The changes in the shock wave intensity in the two gas wave tubes with time are shown in Figure 9. Under the action of the wave elimination chamber, the reflected shock wave in the gas wave tube was significantly weakened; it can be seen that the Helmholtz gas wave tube $\mathrm{P} / \mathrm{P}_{\text {os }}$ value was only 1.7 , and the $\mathrm{P} / \mathrm{P}_{\mathrm{os}}$ value of the traditional gas wave tube was 1.9. The reflected shock wave intensity was reduced by about $11 \%$.

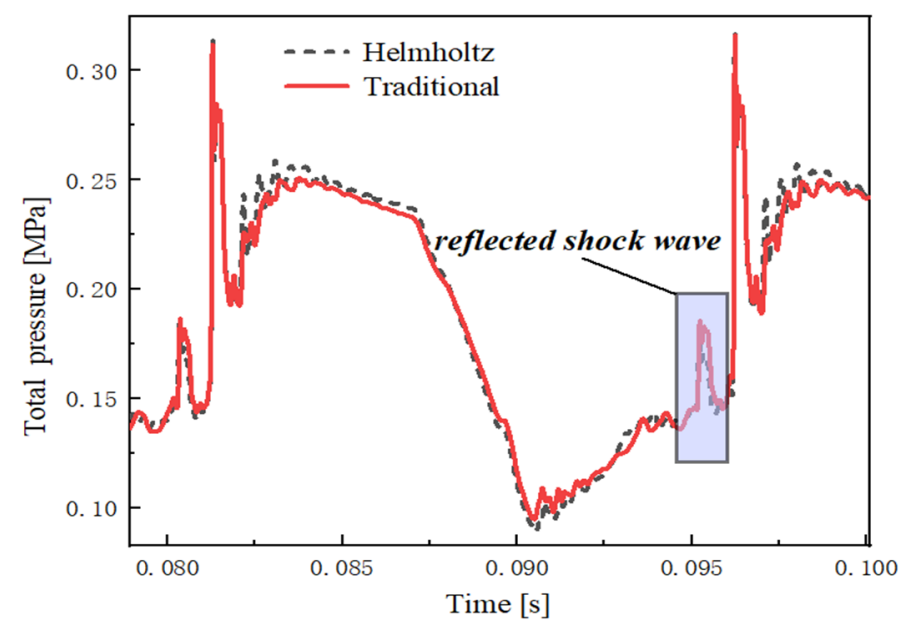

Figure 9. The curve of the shock wave intensity in the two gas wave tubes at the front end of the chamber versus time.

Figure 10 shows the gas mass weighted average total temperature $T_{\mathrm{av}}$ with time for the two gas wave tube structures in a cycle where the jet duty ratio was $1 / 2$, the expansion ratios $\varepsilon$ were 2,3 , and 4 , and the jet frequencies $\mathrm{f}$ were $25 \mathrm{~Hz}$ and $80 \mathrm{~Hz}$. It can be seen from the figure that the jet gas in the exhaust stage is expanded and cooled by the original gas in the compression tube and then discharged from the outlet to obtain a lower-temperature refrigerant gas. From Figure 10a,b, it can be seen that the cooling temperature of the 
Helmholtz central wave-elimination chamber was significantly lower than that of the gas wave tube of the traditional wave-elimination chamber.

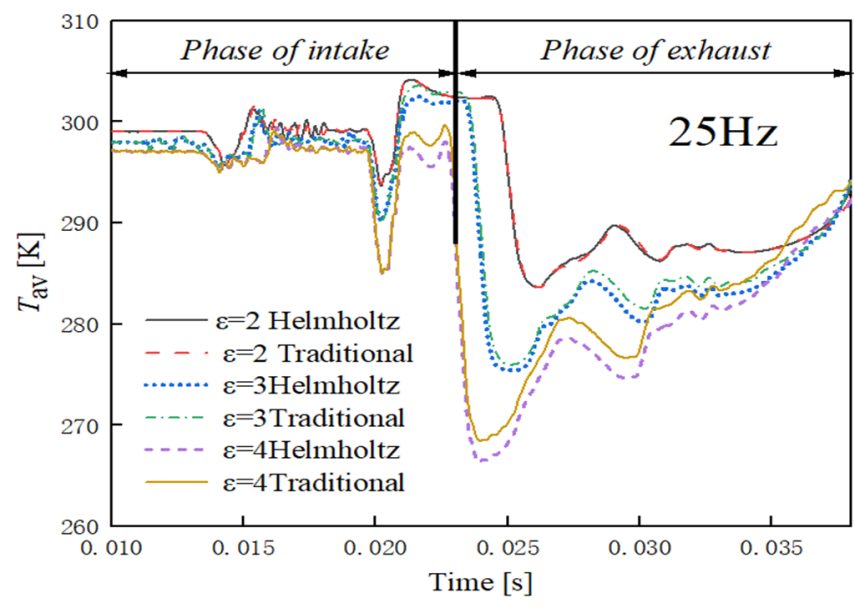

(a)

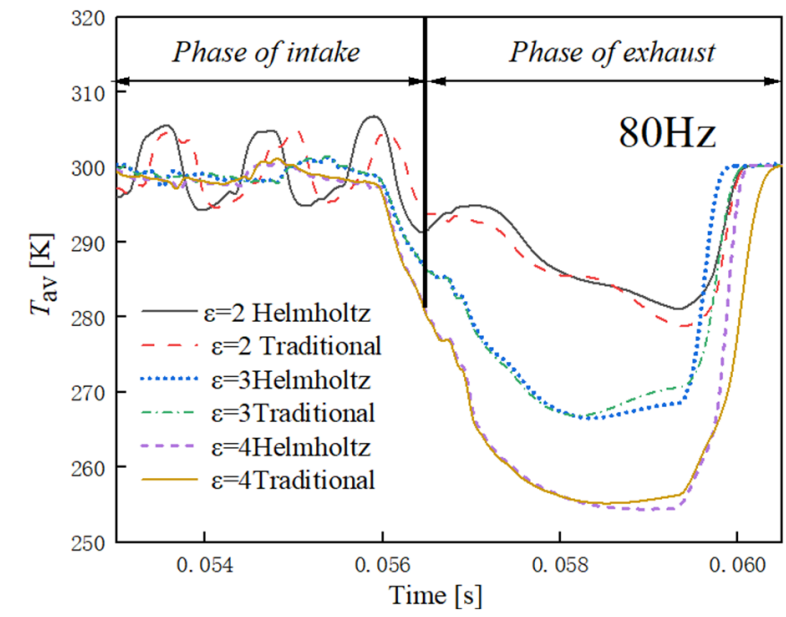

(b)

Figure 10. The variation in the total temperature $T_{\mathrm{av}}$ of the two gas wave tube structures with time in one cycle: (a) $25 \mathrm{~Hz}$; (b) $80 \mathrm{~Hz}$.

Figure 11 shows the numerical simulation of the refrigeration efficiency of the Helmholtz wave-elimination chamber and the traditional wave-elimination chamber as a function of the ejection frequency. Under the condition where the jet duty ratio was $1 / 2$ and the expansion ratios $\varepsilon$ were 2,3 , and 4 , the refrigeration efficiency increased by about $11-18 \%$ with the frequency conversion; the refrigeration efficiency showed a relative increase of about $2.9 \%(50 \mathrm{~Hz}), 2 \%(40 \mathrm{~Hz})$, and $2.2 \%(25 \mathrm{~Hz})$. In addition, due to the increase in the expansion ratio, the turbulence intensity, the temperature difference, and the pressure gradient in the tube, the efficiency was generally slightly improved. Furthermore, because the temperature in the tube was higher, the wave speed increased. The corresponding efficiency extremes were also improved with the increase in the ejection frequency.



Figure 11. The refrigeration efficiency of the two types of gas wave tubes numerically simulated with the change of the ejection frequency.

Through the numerical simulation method, combined with the structural calculation of the pulsating jet and the gas wave tube, it was shown that the Helmholtz-type gas wave tube reduces the loss of incident kinetic energy and increases the consumption of reflected 
shock waves. The reflected shock wave is reflected multiple times between the middle chamber and the end of the gas wave tube, which weakens the intensity of the reflected shock wave and improves the refrigeration efficiency of the gas wave tube.

\section{Experimental Study and Comparison of Two Kinds of Gas Wave Tube Refrigeration Efficiency}

We constructed a single-tube gas wave refrigerator to verify the influence on the refrigeration efficiency of the two gas wave tube structures. The exhaust gas of the single-tube small-volume gas wave refrigerator is easily affected by the temperature of the surrounding environment, making it difficult to accurately measure the exhaust temperature. However, if the changing law of environmental influence factors is observed, the contrasting values of different air wave tube structure refrigeration efficiencies are easy to measure and distinguish; hence, it is a more economical and effective experimental research method.

\subsection{Experimental Facilities}

The experimental device is shown in Figure 12. The single-tube experimental machine was built by accurately aligning the jet nozzle with the opening of the single air wave tube and then fixing it. Between the nozzle and the gas wave tube orifice, a jet pulse modulation disc with two symmetrical and transparent arc openings was inserted. When the arc opening turns to the nozzle and the opening position of the gas wave tube, air is ejected into the gas wave tube. During the exhaust phase, the gas wave tube entrance is blocked by the pulse modulation disc, and the air flows out from the front end of the gas wave tube toward the outlet. According to the requirements of intake duty ratio $1 / 2$ and duty ratio $1 / 4$, the ratio of the transparent arc length to the closed arc length of the pulse modulation disc was 1:1 and 1:2, respectively, as shown in Figure 13.

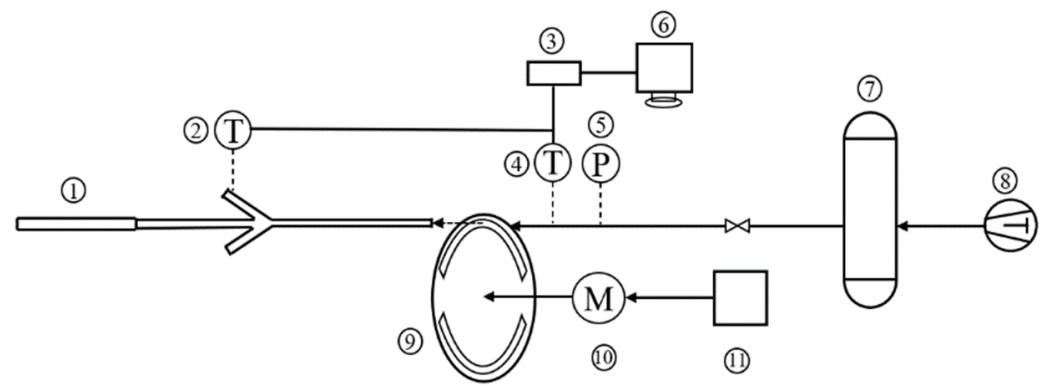

Figure 12. Schematic diagram of the experimental process. 1-gas wave tube; 2, 4-temperature sensor; 3-PLC (Programmable Logic Controller); 5-pressure gauge; 6-computer; 7-air tank; 8-air compressor; 9-pulse jet modulation disc; 10-motor; 11-inverter.

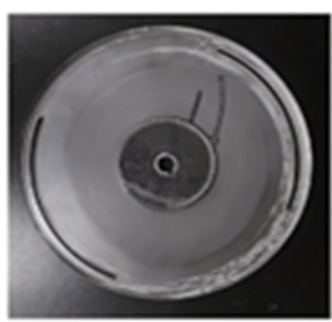

(a)

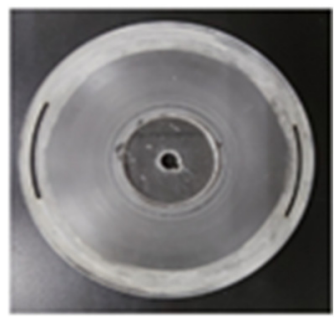

(b)

Figure 13. Pulse modulation discs with different duty cycles: (a) $1 / 2$ duty cycle; (b) $1 / 4$ duty cycle.

The pulse modulation disc was driven by an AC motor whose speed was regulated by a frequency converter. The frequency $\mathrm{f}$ was changed by adjusting the motor speed $\mathrm{n}$ $(\mathrm{r} / \mathrm{min})$. Because there were two arc-shaped openings on the disc, $\mathrm{f}=2 \mathrm{n} / 60 \mathrm{~Hz}$. The outlet structure adopted an acrylic plate to engrave the flow channel; the gas wave tube was 
copper tube, the front tube length was $2000 \mathrm{~mm}$, and the inner diameter was $10 \mathrm{~mm}$. The distance between the two chambers of the two airwave tubes was $1000 \mathrm{~mm}$, and the inner diameter of the tube was $10 \mathrm{~mm}$. The inner diameter of the chamber was about $96 \mathrm{~mm}$, and the length of the inner chamber was about $100 \mathrm{~mm}$. There are three types of wave-elimination chamber. The first two are hollow cylinders made of aluminum. The difference between the two is whether both ends are open. These three structures are shown in Figure 14. The Helmholtz wave-elimination chamber proposed in this paper was made of Plexiglass.

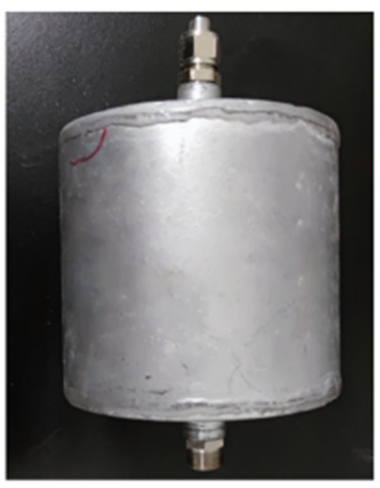

(a)

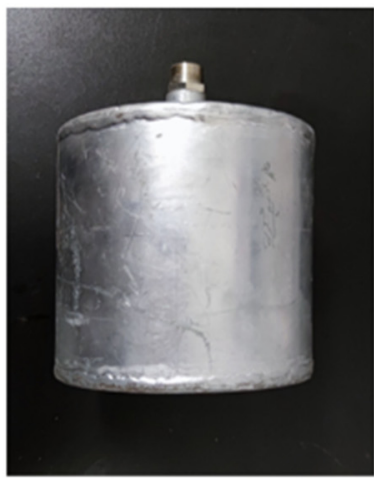

(b)

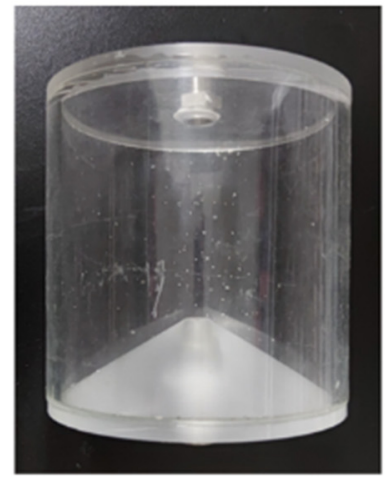

(c)

Figure 14. Different types of chambers: (a) traditional chamber; (b) end wave chamber; (c) Helmholtz chamber.

\subsection{Experimental Results and Analysis}

In the actual experiment, the ambient temperature was uncontrollable and was different from the idealized instantaneous ejection into the gas wave tube and instantaneous exhaust used in the simulation. For a certain distance between the nozzle and the gas wave tube opening, the jet caused a certain degree of loss before entering the gas wave tube, which, along with other complications, made the refrigeration efficiency obtained by the experiment lower than the value obtained by the simulation.

The pressure sensor (5) was inserted into the air supply pipe close to the nozzle to measure the total pressure $P_{\text {in }}$ at the inlet of the air wave pipe. The valve was used to adjust the pressure to 0.1 and $0.2 \mathrm{MPa}$. The exhaust static pressure was the atmospheric pressure, and the corresponding expansion ratios $\varepsilon$ were close to 2 and 3 , respectively. A temperature sensor (4) was also inserted into the air supply pipe close to the nozzle for measurement, which was used as the total intake temperature $T_{\text {out }}$. For the measurement of the cooling temperature of the outlet air, because the modulation disc 9 was attached to the arc-shaped groove on the end of the gas wave tube, the pulse exhaust and reflection caused by the speed change made it difficult to accurately measure the exhaust temperature in the edge area of the pulse jet modulation disc. Therefore, this experiment adopted an indirect method that involved setting a flow channel near the opening of the gas wave tube, inserting a temperature sensor (2), measuring the time-average temperature $T_{\mathrm{a}}$ near the opening, and then approximately converting to the exhaust temperature $T_{\text {out }}$. Although the accurate refrigeration temperature cannot be measured in this way, it is clear enough that the measurement results of uniform conditions can be used to compare the refrigeration efficiency of various gas wave tubes.

Because the room temperature injection gas and the refrigerating gas alternately pass through the opening of the gas wave tube, and the ambient gas outside the metal gas wave tube heats the small amount of refrigerating gas, the time-average temperature $T_{a}$ measured at the opening of the gas wave tube is much higher than the actual cooling discharge temperature $T_{\text {out }}$. Regardless of the influence of environmental heating, it is assumed that the heat transfer $Q=m c_{p} \Delta T$ of the ejection and exhaust gas to the sensor are equal. Assuming that the specific heat capacity $c_{\mathrm{p}}$ is constant, and the mass $\mathrm{m}$ of injection 
and exhaust gas is equal, the following equations are given according to the relationship of equal heat transfer: $T_{\text {in }}-T_{\mathrm{a}}=T_{\mathrm{a}}-T_{\text {out }}$ and $T_{\text {out }}=2 T_{\mathrm{a}}-T_{\mathrm{in}}$. The refrigeration efficiency values of different gas wave tube structures under different ejection conditions and frequency conditions can be calculated by substituting the calculated exhaust temperature $T_{\text {out }}$, the measured total intake temperature $T_{\mathrm{in}}$, and the expansion ratio $\varepsilon$ into Equation (2).

In fact, corresponding to the improved efficiency of the new gas wave tube, the ambient gas outside the tube had a greater impact on the refrigerant gas inside the tube, and the heating was also stronger. However, since the above simplified formula is not considered, the calculated efficiency curve gap between the two gas wave tubes is smaller than the actual value. In addition, the heating coefficient of the nozzle with the temperature difference is difficult to estimate, so it was not considered. This leads to a certain error in the results between the actual experiment and the numerical simulation under ideal boundary conditions; however, it is still accurate enough to compare the refrigeration efficiency of the two gas wave tubes.

Figure 15a shows the cooling efficiency obtained from the measured time-average temperature at the exit section of the gas wave tube of the two structures under the condition where the jet duty ratio was $1 / 2$ and the expansion ratios $\varepsilon$ were 2 and 3 . With the increase in frequency, the cooling efficiency also increased by $2 \%$ to $6 \%$; the Helmholtz chopper chamber increased by about $3.5 \%(50 \mathrm{~Hz})$ and $3.7 \%(90 \mathrm{~Hz})$ compared with the gas wave tube of the traditional middle chopper chamber. In addition, the efficiency extreme value increased with the fluctuation of the ejection frequency. Figure 15b shows the cooling efficiency obtained by the measured time-average temperature at the exit section of the gas wave tube of the two structures under the condition where the jet duty ratio was $1 / 4$ and the expansion ratios $\varepsilon$ were 2 and 3. The refrigeration efficiency also increased by $2 \%$ to $5 \%$. The Helmholtz central wave-elimination chamber and the gas wave tube of the traditional central wave-elimination chamber were relatively improved by about $5 \%(50 \mathrm{~Hz})$ and $2 \%$ $(90 \mathrm{~Hz})$, respectively.

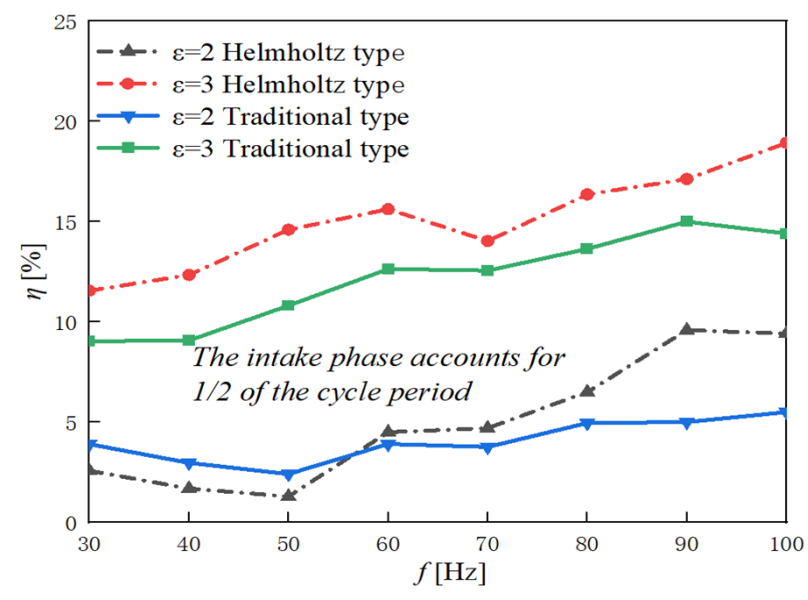

(a)

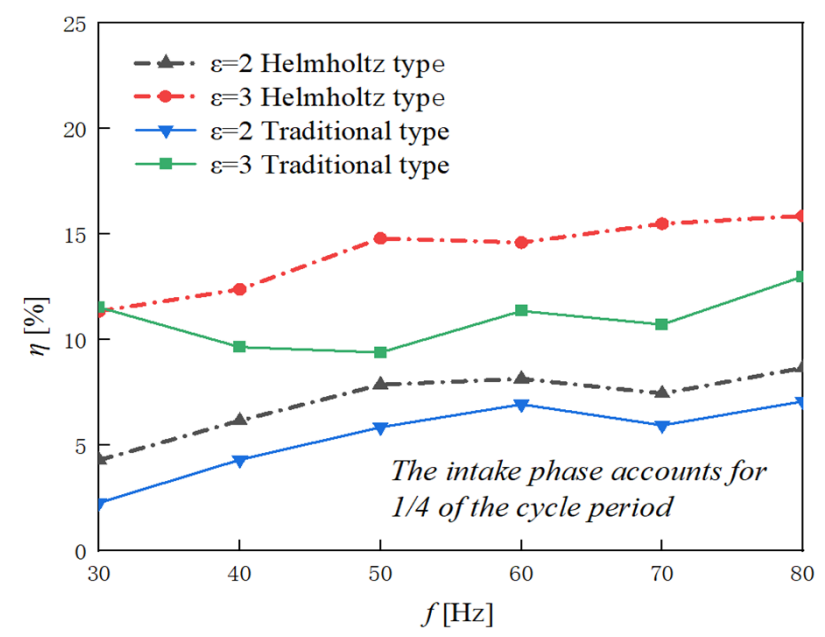

(b)

Figure 15. Refrigeration efficiency change diagram with frequency: (a) $1 / 2$ duty cycle; (b) $1 / 4$ duty cycle.

It can be seen from the experimental results that the refrigeration efficiency and flatness of the Helmholtz wave-elimination chamber gas wave tube were significantly higher than that of the existing traditional wave-elimination chamber gas wave tube. The ejection frequencies corresponding to the peak and trough efficiency of the two kinds of gas wave tubes in the experiment were also basically consistent with the simulation results. 


\section{Conclusions}

The gas wave tube structure of the static gas wave refrigerator was optimized by the method of combining CFD numerical simulation and experimental studies. The shock wave absorption chamber was changed from a traditional chamber to a Helmholtz chamber. The refrigeration efficiencies of the two gas wave tube structures with varying jet frequencies when the expansion ratio was 2, 3, and 4 were compared and analyzed. The results showed that:

(1) Through chamber flow field analysis and CFD three-dimensional numerical simulation, it was shown that Helmholtz-type wave-elimination chamber reduced forward shock wave dissipation. The collision between the incident airflow and the chamber wall of a traditional wave-breaking chamber consumes a great deal of kinetic energy, resulting in a total pressure retention rate much lower than that of the Helmholtz wave-breaking chamber. The use of Helmholtz-type chamber increased the total pressure retention rate by $7 \%$.

(2) Through the two-dimensional numerical simulation method, combining the pulsating ejection gas and the gas wave tube structure, it was shown that the Helmholtz gas wave tube reduced the incident kinetic energy loss, weakened the reflected shock wave, and improved the cooling efficiency. The intensity of the reflected shock wave was reduced by about $11 \%$. In the three expansion ratios, the relative increase in refrigeration efficiency was about $2.9 \%(50 \mathrm{~Hz}), 2 \%(40 \mathrm{~Hz})$, and $2.2 \%(25 \mathrm{~Hz})$.

(3) A single-tube gas wave machine model was used to conduct a comparative experimental study on the efficiency of the two gas wave tube structures. Although the experimental model structure itself had uncontrollable ambient temperature and the loss of gas at the inlet, the overall efficiency was very low. It can be clearly seen from the comparison of the gas wave tube structures that the refrigeration efficiency of the Helmholtz-type gas wave tube increased significantly, and the difference in the trend of the refrigeration efficiency range of the two kinds of gas wave tubes corresponded to the peak and valley values of the efficiency. Although the ejection frequency was more consistent with the simulation results, the refrigeration efficiency of the gas wave tube was significantly increased.

Author Contributions: Conceptualization, W.N.; methodology, W.N.; software, W.N.; validation, W.N., X.L. and P.Y.; formal analysis, J.Z.; investigation, W.N.; resources, D.H.; data curation, W.N.; writing-original draft preparation, W.N.; writing—review and editing, X.L.; supervision, D.H.; project administration, X.L.; funding acquisition, D.H. All authors have read and agreed to the published version of the manuscript.

Funding: This work was supported by the National Key Research and Development Program of China (2018YFA0704601) and the Natural Science Foundation of China (21776029).

Institutional Review Board Statement: Not applicable.

Informed Consent Statement: Not applicable.

Data Availability Statement: Not applicable.

Acknowledgments: The authors thank the opportunity and financial support to carry on this project from the Natural Science Foundation of China.

Conflicts of Interest: The authors declare no conflict of interest.

\section{References}

1. Marchal, P.; Malek, S.; Vitard, J.C. Skid-mounted rotating thermal separator. Oil Gas 1984, 11, 55-58.

2. Yu, H.-R. Flow in heat separator. J. Dalian Univ. Technol. 1984, 23, 1-7.

3. Peng, X.-H.; Zhong, H.-G. Application of gas wave refrigerator in simulated altitude test. Gas Turbine Exp. Res. 2012, 25, 27-30.

4. Liu, P.-Q.; Xu, S.-Y.; Wang, Z.-W.; Liu, S.; Hu, D.; College of Chemical Engineering, Dalian University of Technolog. Influence of offset angle on refrigeration efficiency of gas wave refrigerator and prediction for optimal offset angle. CIESC J. 2014, 65, 4271-4277. 
5. Zheng, M.-F.; Liu, X.; Huang, C.; Lin, Y.; Lei, X.; Li, X.; College of Chemistry and Chemical Engineering, Fuzhou University. Incident shock wave attenuation in oscillatory tube and influence on performance of pressure wave refrigerator. CIESC J. 2014, 65, 3410-3417.

6. Li, Z.-C.; Xu, L. Research and application of gas wave refrigerator. Cryogenics 2002, 126, $22-27$.

7. Yu, Y.; Liu, P.-Q.; Wang, Y.-L.; Leng, C.; Zhao, Y.; Wang, J.; Hu, D.; School of Chemical Machinery, Dalian University of Technology. Flow and thermodynamic properties of efficient gas wave refrigeration plant. CIESC J. 2017, 68, 3039-3048.

8. Fang, Y.-Q.; Zheng, J.; Liu, R.-J.; Zhu, C.; Fan, J.; Hu, D.-P. Experimental investigation of influence factor of gas wave refrigeration efficiency. Aerodyn. Exp. Meas. Control. 1993, 7, 15-18.

9. Hu, D.-P.; Liu, P.-Q.; Zhu, C. A weakening reflected shock wave method in oscillating tube. CIESC J. 2008, 59, 562-566.

10. Zou, J.-P.; Liu, X.-W.; Chen, S.-H. Experiment about weakening the energy of the reflecting shock waves in the incepting tube. Cryogenics 2001, 121, 48-53.

11. Liu, X.-W.; Zou, J.-P.; Chen, S.-H.; Jin, L.-A. Study on the influence of gas wave tube end boundary condition to refrigeration efficiency. Fluid Mach. 2001, 29, 55-57.

12. Li, X.-L.; Zhu, C. Elimination of reflect shock wave in oscillatory tube. CIESC J. 2001, 52, 379-380.

13. Dai, Y.-Q.; Hu, D.-P.; Liu, W.; Zhu, C. The CFD analysis of oscillatory tube equipped with compound damps. Low Temp. Spec. Gases 2003, 21, 23-24.

14. Zhu, X.-Q. Study on the optimum length of the accept tube of gas wave refrigerator. J. Wuxi Inst. Light Ind. 1993, 12, 135-141.

15. $\mathrm{Li}, \mathrm{X}$.-L. Experimental study on influences of the tube length on cooling effects of the oscillating tube. Refrigeration 1993, 2, 15-18.

16. Liu, P.-Q.; Hu, D.-P.; Zhao, W.-J.; Zhu, C.; Dai, Y.; Liu, R.; Zou, J. Numerical prediction for extreme jet flow frequency of rotary gas wave refrigerator. CIESC J. 2009, 60, 1-5.

17. Al-Obaidi, A.R. Study the influence of concavity shapes on augmentation of heat-transfer performance, pressure field, and fluid pattern in three-dimensional pipe. Heat Transf. 2021, 50, 4354-4381. [CrossRef]

18. Al-Obaidi, A.R.; Alhamid, J.; Hamad, F. Flow felid and heat transfer enhancement investigations by using a combination of corrugated tubes with a twisted tape within 3D circular tube based on different dimple configurations. Heat Transf. 2021, 50, 6868-6885. [CrossRef]

19. Al-Obaidi, A.R. Investigation of the flow, pressure drop characteristics, and augmentation of heat performance in a $3 \mathrm{D}$ flow pipe based on different inserts of twisted tape configurations. Heat Transf. 2021, 50, 5049-5079. [CrossRef]

20. Li, D.; Kang, Y.; Ding, X.-L.; Wang, X.-C.; Fang, Z.-L. Effects of area discontinuity at nozzle inlet on the characteristics of high speed self-excited oscillation pulsed waterjets. Exp. Therm. Fluid Sci. 2016, 79, 254-265. [CrossRef]

21. Fang, Z.-L.; Zheng, F.-D.; Xiong, T.; Wei, W.; Jiang, P.; Wu, Q.; Wang, Y.; Fei, Y. Large eddy simulation of self-excited oscillation inside Helmholtz oscillator. Int. J. Multiph. Flow 2020, 126, 103253. [CrossRef]

22. Xue, X.; Yu, Y.; Zhao, J. Study on draining off water mechanism and interaction characteristic of high-temperature and highpressure combustion-gas jets with the water. Appl. Therm. Eng. 2018, 143, 570-581. [CrossRef]

23. Wang, F.-J. Computational Fluid Dynamics Analysis: The Principle and Application of CFD Software; Tsinghua University Press Co., Ltd.: Beijing, China, 2014.

24. Deconinck, H.; Roe, P.L.; Struijs, R.A. multidimensional generalization of Roe's flux difference splitter for the Euler equations Comput. Fluids 1993, 22, 215-222. [CrossRef]

25. Saito, T.; Voinovich, P.; Zhao, W.; Shibasaki, K.; Shibasaki, S.; Takayama, K. Experimental and numerical study of pressure wave refrigerator performance. Shock. Waves 2003, 13, 253-259. [CrossRef] 